[11] R. Engelking and A. Pełczyński, Remarks on dyadic spaces, Coll. Math. 11 (1963), pp. 55-63.

[12] A.S. Esenin-Volpin, On the relation between the local and integral weight in dyadic bicompacta, Dokl. Akad. Nauk SSSR 68 (1949), pp. 441-444.

[13] F. Galvin and B. Jonsson, Distributive sublattices of a free lattice, Canad. J. Math. 13 (1961), pp. 265-272.

[14] J. Gerlits, On a problem of S. Mrówka, Period. Math. Hung. 4 (1973), pp. 71-79.

[15] - On a generalization of dyadicity, Studia Sci. Math. Hung. 13 (1978), pp. 1-17.

[16] J. de Groot, Supercompactness and superextensions, in Contributions to extension theory of topological structure, Symp. Berlin 1967, Deutscher Verlag Wiss., Berlin 1969, pp. 89-90

[17] I. Ju hasz, Cardinal Functions in Topology - Ten Years later, Mathematical Centre Tracts 123, Amsterdam 1980.

[18] R. H. Marty, m-adic spaces, Acta Math. Acad. Sci. Hung. 22 (1971), 441-447.

[19] S. Mrówka, Mazur Theorem and m-adic spaces, Bull. Acad. Polon. Sci. 43 No. 6 (1970), pp. 299-305.

[20] I. I. Parovicenko, A universal bicompactum of weight $\$$, Soviet Math. Dokl. 4 (1963), pp. 592-595.

[21] N. A. Sanin, On the product of topological spaces, Trudy Mat. Inst. Steklov, 24 (1948),

22] L. B. Sapiro, The space of closed subsets of $D^{\mathrm{N}_{2}}$ is not a dyadic bicompact, Soviet Math. Dokl. 17 (1976), pp. 937-941.

[23] S. Sirota, Spectral representation of spaces of closed subsets of bicompacta, Soviet Math. Dokl. 9 (1968), pp. 997-1000.

DEPARTMENT OF MATHEMATICS

THE UNIVERSITY OF MANITOBA

Winnipeg, Manitoba

Canada R3T 2N2

\section{On the transformers of the Zahorski classes of functions}

by

\section{Grzegorz Krzykowski (Gdańsk)}

Abstract. Let $\mathscr{M}_{i}(i=1, \ldots, 5)$ denote the Zahorski classes of functions defined on interval $I=(0,1)$. By $H_{M_{L}}$ we denote the set of all increasing homeomorphism $h$ of interval $I$ onto itself which leaves $\mathscr{M}_{i}$ invariant, i.e., such that for every $f \in \mathscr{M}_{i} f \circ h \in \mathscr{M}_{i}$. In this paper it is shown that there are proper inclusion $H_{\Delta H_{i}} \supset H_{\mathcal{M}_{t+1}}$, for $i=1,2,3$. We give a characterization of the class $H_{\mathscr{M}_{4}}$ and the examples of homeomorphisms showing that classes $H_{\mathcal{H}_{5}} \backslash H_{\mathcal{M}_{4}}$ and $H_{\mathcal{M}_{4}} \backslash H_{\mathcal{M}_{5}}$ are nonempty. We also establish the inclusion $H_{\mathcal{H}_{4}} \supset H_{b \Delta}$ where $H_{b \Delta}$ is the class of homeomorphisms preserving bounded derivatives.

Let $g$ be a homeomorphism of $(0,1)$ onto itself, $g(0)=0, g(1)=1$. If $\mathscr{F}$ is an arbitrary family of real functions defined on $(0,1)$ then $g$ is said to be a transformer on $\mathscr{F}$ if $f \circ g \in \mathscr{F}$ holds for every $f \in \mathscr{F}$. Let $H_{\mathscr{F}}$ denote the class of transformers of $\mathscr{F}$.

M. Laczkovich and G. Petruska in [7] have given a necessary and sufficient condition for $g$ to be a transformer on a class of derivatives.

In 1950, Z. Zahorski [9] considered a hierarchy of classes of functions, $\mathscr{M}_{k}$, $i=0, \ldots, 5$. The largest class, $\mathscr{M}_{0}$, turned out to be Darboux and first class of functions: the smallest, $\mathscr{M}_{5}$, turned out to be class of approximately continuous functions. He showed how the classes of derivatives and bounded derivatives fit into the scheme.

In the present paper we study a hierarchy of classes $H_{\mathbb{A}_{k}}, k=0, \ldots, 5$ (for the sake of simplicity we use the notation $\left.H_{k}, k=0, \ldots, 5\right)$ and consider a number of related properties.

Throughout this paper the word "set" means a Lebesgue measurable subset of open interval $(0,1)$, the word "homeomorphism" means an increasing homeomorphism of $(0,1)$ onto itself. By a "function" we means a real function on $(0,1)$. $A^{\prime}=(0,1) \backslash A,|A|$ being the Lebesgue measure of the set $A$ and $d(A, a)$ being the density of the set $A$ in the point $a$. As usual, $d^{+}(A, a)$ and $d^{-}(A, a)$ denote the righthand side and lefthand side densities and $d(A, a)$ the upper density.

We begin with the definition of the Zahorski classes of sets.

Definition 1 . Let $E$ be a nonempty set of type $F_{\sigma}$. We say $E$ belongs to class: 
$M_{0}$ if every point of $E$ is a point of bilateral accumulation of $E$;

$M_{1}$ if every point of $E$ is a point of bilateral condensation of $E$;

$M_{2}$ if each one-side neighbourhood of each $x \in E$ intersects $E$ in a set of positive measure;

$M_{3}$ if for each $x \in E$ and each sequence $\left\{I_{n}\right\}_{n=1}^{\infty}$ of closed intervals converging to $x$ and not containing $x$ such that $\left|I_{n} \cap E\right|=0$ for each $n$, we have

$$
\lim _{n \rightarrow \infty} \frac{\left|I_{n}\right|}{\operatorname{dist}\left(x, I_{n}\right)}=0 \text {; }
$$

$M_{4}$ if there exist a sequence of closed sets $\left\{F_{n}\right\}_{n=1}^{\infty}$ and a sequence of positive numbers $\left\{\eta_{n}\right\}_{n=1}^{\infty}$ such that $E=\bigcup_{n=1}^{\infty} F_{n}$ and for $x \in F_{n}$ and each $c>0$ there exists a number $\varepsilon(x, c)=0$ such that if $h$ and $h_{1}$ satisfy $h h_{1}>0, h / h_{1}<c,\left|h+h_{1}\right|<\varepsilon(x, c)$, then

$$
\frac{\left|E \cap\left(x+h, x+h_{1}\right)\right|}{\left|h_{1}\right|}>\eta_{n}
$$

$M_{5}$ if every point of $E$ is a point of density of $E$.

DEFINITION 2. Let $f$ be real function defined on an interval $I=(0,1)$, $k=1,2, \ldots, 5$. We say $f$ is in class $\mathscr{M}_{k}$ if every associated set of $f$ is in $M_{k}$. The associated sets of $f$ are all sets of the from $E_{\alpha}(f)=\{x: f(x)<\alpha\}$ or $E^{\alpha}(f)$ $=\{x: f(x)>\alpha\}$, for $\alpha \in R$.

Definition 3. Let $H_{i}, i=1, \ldots, 5$, denote the class of all homeomorphisms $h$ such that for all $f \in \mathscr{M}_{i}, f \circ h \in \mathscr{M}_{i}$.

LEMMA 1. $h \in H_{i}$ if and only if for each $A \in M_{i} h^{-1}(A) \in M_{i}(i=1, \ldots, 5)$,

Proof. Let $h$ be a homeomorphism such that for every $A \in M_{i}, h^{-1}(A) \in M_{i}$. Let $f \in \mathscr{M}_{i}$. Observe that $E_{\alpha}(f \circ h)=h^{-1}\left(E_{\alpha}(f)\right)$. Since $E_{\alpha}(f) \in M_{i}, h^{-1}\left(E_{\alpha}\left(f^{\dagger}\right)\right) \in M_{i}$. Thus, $E_{\alpha}(f \circ h) \in M_{i}$. In the similar way we may prove that $F_{\alpha}(f \circ h) \in M_{i}$. Thus, we see that $h \in H_{i}$. Let $h \in H_{i}$ and $A \in M_{i}$. Then there exists a function $f \in \mathscr{A}_{i}$ such that $0<f(x)<1$ for all $x \in A$, and $f(x)=0$ for all $x \notin A$ (see [1]). Therefore $E^{0}(f \circ h)$ $=h^{-1}(A)$. Then $h^{-1}(A) \in M_{i}$

We are now ready to establish inclusion relationships for $H_{i}, i=1, \ldots, 5$.

THEOREM 1. The following proper inclusions holds: $H_{1} \supset H_{2} \supset H_{3} \supset H_{4}$

Proof. Theorems 1.1 ([3], p. 9) and 3.5 ([3], p. 16) show that $\mathscr{M}_{0}=\mathscr{M}_{1}$ and that $H_{1}$ is the set of all homeomorphisms. Let $h \notin H_{2}$, and $g=h^{-1}$. By Lemma 1 there exists a set $A \in M_{2}$, such that $g(A) \notin M_{2}$. Then, there exist $y \in g(A)$ and $\eta>0$ such that $|(y, y+\eta) \cap g(A)|=0$ or $|(y-\eta, y) \cap g(A)|=0$. Suppose that $|(y, y+\eta) \cap g(A)|=0$ (in some way, we obtain the result for the second condition). Let $x=g^{-1}(y)$ and $x+\varepsilon=g^{-1}(y+\varepsilon)$. Since $A \in M_{2},|(x, x+\varepsilon) \cap A|>0$. Let $B=\{t: t \in(x, x+\varepsilon) \cap A$ and $d(A, t)=1\}$. Let $B^{*}$ denote the set of type $F_{\sigma}$, such that $B^{*} \subset B$ and $\left|B^{*}\right|=|B|$. Thus $B^{*} \in M_{5}$ and also $B^{*} \in M_{3}$. Since $\left|g\left(B^{*}\right)\right|$ $=0, h \notin H_{3}$. This completes the proof of the inclusion $H_{2} \supset H_{3}$.
Let us now focus our atterition on the inclusion $H_{3} \supset H_{4}$. The proof is similar to that of the inclusion $\mathrm{H}_{2} \supset \mathrm{H}_{3}$.

Suppose that $h \notin H_{3}$. By Lemma 1 there exists a set $A \in M_{3}$, such that $g(A) \notin M_{3}$ $\left(g=h^{-1}\right)$. Then, there exists $y \in g(A)$ and a sequence of closed intervals $\left\{I_{n}\right\}_{n=1}^{\infty}$ converging to $y$, such that $\left|I_{n} \cap g(A)\right|=0$ and $\left|I_{n}\right|>c \operatorname{dist}\left(y, I_{n}\right)$, where $c>0$ $(n=1,2, \ldots)$. Suppose that $x=g^{-1}(y), J_{n}=g^{-1}\left(I_{n}\right),(n=1,2, \ldots)$. We may assume that for all $n \in N\left|A \cap J_{n}\right|=0$ (if there exists $n_{0} \in N$ such that $\left|A \cap J_{n_{0}}\right|>0$, then there exists a set $A^{*}$ such that $A^{*} \in M_{5}$ and $\left|g\left(A^{*}\right)\right|=0$, then by Lemma 1 $\left.g^{-1} \notin H_{4}\right)$. Since $A \in M_{3}$, we have

$$
\lim _{n \rightarrow \infty} \frac{\left|J_{n}\right|}{\operatorname{dist}\left(x, J_{n}\right)}=0 \text {. }
$$

Let $B=(0,1) \backslash \bigcup_{n=1}^{\infty} J_{n}$. We have $B \in M_{3}$. In the same way we obtain the result for a set $E=(0,1) \backslash \bigcup_{k=1}^{\infty} J_{n_{k}}$, where $\left\{J_{n_{k}}\right\}_{k=1}^{\infty}$ is a subsequence of the sequence $\left\{J_{n}\right\}_{n=1}^{\infty}$. Choose a subsequence $\left\{J_{n_{k}}\right\}_{k=1}^{\infty}$ of sequence $\left\{J_{n}\right\}_{n=1}^{\infty}$ such that $d\left(\bigcup_{k=1}^{\infty} J_{n_{k}}, x\right)=0$. Let $E=(0,1) \backslash \bigcup_{k=1}^{\infty} J_{n_{k}}$. Since $d(E, x)=1$, the set $E \in M_{5}$. It follows that $E \in M_{4}$ and $g(E) \notin M_{3}$. Thus $h \notin H_{4}$. This completes the proof of all the inclusions.

We now prove that the inclusions are proper. We construct three examples. Let the homeomorphism $g_{1}$ map some set of positive measure onto a set of measure zero. Thus $h_{1}=g_{1}^{-1} \notin H_{2}$.

Let $A=(0, x] \cup \bigcup_{n=1}^{\infty} J_{n}$, where $J_{n}=\left(a_{n}, b_{n}\right), d(A, x)=1, x \in(0,1), x<a_{n+1}$ $<b_{n+1}<a_{n}<b_{1}=1$. Let $B=(0, x] \cup \bigcup_{n=1}^{\infty} I_{n}$, where $I_{n}=\left(a_{n}^{\prime}, b_{n}^{\prime}\right), x<a_{n+1}^{\prime}<b_{n+1}^{\prime}$ $<a_{n}^{\prime}<b_{1}^{\prime}=1$ and

$$
\lim _{n \rightarrow \infty} \frac{\left|\left(b_{n+1}^{\prime}, a_{n}^{\prime}\right)\right|}{b_{n+1}^{\prime}}=\alpha>0
$$

Define the homeomorphism $g_{2}$ by the formula $g_{2}(t)=t$ for all $t \in(0, x]$, $g_{2}\left(a_{n}\right)=a_{n}^{\prime}, g_{2}\left(b_{n}\right)=b_{n}^{\prime}(n=1,2, \ldots)$, and $g_{2}$ is linear on each interval $\left(a_{n}, b_{n}\right)$ or $\left(b_{n+1}, a_{n}\right)(n=1,2, \ldots)$. The proof that $h_{2}=g_{2}^{-1} \in H_{2} \backslash H_{3}$ follows immediately from Lemma 1.

We now show that $H_{3} \neq H_{4}$. Let

$$
E=(0, x] \cup \bigcup_{n=n_{0}}^{\infty}\left(x+\frac{1}{n}, x+\frac{1}{n}+r_{n}\right),
$$

where

Let

$$
x \in(0,1), \quad 1 / n(n-1)>r_{n}>0, \quad d(E, x)=1 .
$$

$A=(0, x] \cap \bigcup_{n=m_{0}}^{\infty}\left(x+\frac{1}{n}, x+\frac{1}{n}+r_{n}^{\prime}\right), \quad \frac{1}{n(n-1)}>r_{n}^{\prime}>0 \quad$ and $\quad d^{+}(A, x)=0$ 
Consequently $A \notin M_{4}$. Define the homeomorphism $g_{3}(t)=t$ for all $t \in(0, x]$, $g_{3}(x+1 / n)=x+1 / n, g_{3}\left(x+1 / n+r_{n}\right)=x+1 / n+r_{n}^{\prime}\left(n=n_{0}, n_{0}+1, \ldots\right)$ and $g_{3}$ is linear on each interval $\left(x+1 / n, x+1 / n+r_{n}\right)$ or $\left(x+1 /(n+1)+r_{n+1}, x+1 / n\right), n=n_{0}$, $n_{0}+1, \ldots$ and $\left(x+1 / n_{0}+r_{n_{0}}, 1\right)$. Thus $d^{+}(A, x)=0$ and $g_{3}(E)=A$, so $h_{3}$ $=g_{3}^{-1} \notin H_{4}$. We now show that $h_{3} \in H_{3}$. Let $D \in M_{3}$, and $\left\{P_{n}\right\}_{n=1}^{\infty}$ be a sequence of closed intervals not containing $x$ and converging to $x$ such that $\left|g_{3}(D) \cap P_{n}\right|=0$, for each $n \in N$. It follows that $\left|D \cap Q_{n}\right|=0$, where $Q_{n}=g_{3}^{-1}\left(P_{n}\right), n=1,2, \ldots$ Since $D \in M_{3}$, we have

$$
\lim _{n \rightarrow \infty} \frac{\left|Q_{n}\right|}{\operatorname{dist}\left(x, Q_{n}\right)}=0
$$

There exists $s=s(n), k=k(n)$ such that where

$$
\inf \left(Q_{n}\right) \in\left[a_{s(n)}, a_{s(n)-1}\right) \quad \text { and } \quad \sup \left(Q_{n}\right) \in\left(a_{s(n)-k(n)+1}, a_{s(n)-k(n)}\right)
$$

We have

$$
\frac{\left|Q_{n}\right|}{\operatorname{dist}\left(x, Q_{n}\right)}=\frac{\left|Q_{n} \cap\left(a_{s}, a_{s-1}\right)\right|}{\operatorname{dist}\left(x, Q_{n}\right)}+\frac{\left|\left(a_{s-1}, a_{s-k+1}\right)\right|}{\operatorname{dist}\left(x, Q_{n}\right)}+\frac{\left|\left(a_{s-k+1}, a_{s-k}\right) \cap Q_{n}\right|}{\operatorname{dist}\left(x, Q_{n}\right)}
$$

Since

we obtain

$$
\lim _{n \rightarrow \infty} \frac{\left|Q_{n}\right|}{\operatorname{dist}\left(x, Q_{n}\right)}=0, \quad \lim _{n \rightarrow \infty} \frac{\left|\left(a_{s-1}, a_{s-k+1}\right)\right|}{\operatorname{dist}\left(x, Q_{n}\right)}=0
$$

$$
\lim _{n \rightarrow \infty} \frac{\left|\left(a_{s(n)-1}, a_{s(n)-k(n)+1}\right)\right|}{a_{s(n)-1}-x}=0
$$

that is, $\lim _{n \rightarrow \infty} k(n) /(s(n)-k(n))=0$. Observe that

$$
n \rightarrow \infty
$$

$$
\frac{\left|P_{n}\right|}{\operatorname{dist}\left(x, P_{n}\right)} \leqslant \frac{\left|\left(a_{s}, a_{s-k}\right)\right|}{a_{s}-x}=\frac{x+\frac{1}{s-k}-x+\frac{1}{s}}{1 / s}=\frac{k}{s-k} .
$$

It follows that $\lim _{n \rightarrow \infty}\left|P_{n}\right| / \operatorname{dist}\left(x, P_{n}\right)=0$, which means that $g_{3}(D) \in M_{3}$ (at the other points of the set $g_{3}(D)$, the definition of the $M_{3}$-set holds, because $g_{3}$ is linear from each side), and the proof of the theorem is complete.

Definition 4 . The $d_{4}$-upper density of the set $X$ at the point $a$ denoted by
$X, a)$ is defined by $d_{4}(X, a)$ is defined by

$$
d_{4}(X, a)=\sup \left\{\lim _{n \rightarrow \infty} \frac{\left|X \cap I_{n}\right|}{\left|I_{n}\right|}\right\}
$$

where supremum is taken for all the sequences of intervals $\left\{I_{n}\right\}_{n=1}^{\infty}$ for with $I_{n} \rightarrow a$ (that is the endpoints of $I_{n}$ converge to $a$ ) and there exists a positive number $c$ such that $\left|I_{n}\right|>c \cdot \operatorname{dist}\left(a, I_{n}\right)(n=1,2, \ldots)$.

LEMMA 2. For every $X \subset(0,1), X$ is $M_{4}$-set if and only if there exists a sequence of closed sets $\left\{F_{n}\right\}_{n=1}^{\infty}$ and a sequence of positive numbers $\left\{\eta_{n}\right\}_{n=1}^{\infty}$ such that $X=\bigcup_{n=1}^{\infty} F_{n}$
and for every $n$ and $x \in F_{n}$ we have $d_{4}\left(X^{\prime}, x\right)<1-\eta_{n}$.

Proof. This is only a reformulation of the definition of $M_{4}$-set (see [6]).

LEMMA 3. For every $X \subset(0,1)$ and $a \in(0,1)$ we have

(i) $0 \leqslant d(X, a) \leqslant d_{4}(X, a) \leqslant 1$

(ii) If $d(X, a)$ exists then $d_{4}(X, a)=d(X, a)$.

The proof of this lemma can be found in [6].

We shall use the following notation ( $\mathrm{p}_{4}$-property for the sets $\left.A, B\right): A \subset_{\mathrm{p}_{4}} B$ means that $A \subset B$ and there exists a sequence $\left\{F_{n}\right\}_{n=1}^{\infty}$ of closed sets and a sequence of positive numbers $\left\{\eta_{n}\right\}_{n=1}^{\infty}$ such that $A=\bigcup_{n=1}^{\infty} F_{n}$ and for every $n$ and $x \in F_{n}$ we
have $d_{4}\left(B^{\prime}, x\right)<1-\eta_{n}$.

DefintTION 5. We say that the homeomorphism $g$ satisfies condition $(\mathrm{H})$ if for every pair of perfect sets $A, B$ such that $A \subset_{\mathrm{p}_{4}} B$, we have $g(A) \subset_{\mathrm{p} 4} g(B)$.

THEOREM 2. A necessary and sufficient condition for $g^{-1} \in H_{4}$ is that $g$ satisfies condition $(\mathrm{H})$.

Proof. Suppose $g$ satisfies condition $(\mathrm{H})$. Let $f \in \mathscr{M}_{4}$. Then there exists a $\mathrm{p}_{4}$-system for $f$ (see [2]), that is, a system of perfect sets $\left\{A_{n}^{y_{r}}, A_{y_{r}}^{n}\right\}, r=1,2, \ldots$, $n=r, r+1, \ldots, y_{1}, y_{2}, \ldots-$ is an enumeration of the rationals, satisfying

$$
\begin{aligned}
& \text { 1. } \quad \bigcup_{n=r}^{\infty} A_{n}^{y_{r}}=E^{y_{r}}(f), \bigcup_{n=r}^{\infty} A_{y_{r}}^{n}=E_{\eta_{r}}(f) \text {, } \\
& \text { 2. } \quad A_{n}^{y_{r}} \subset_{\mathrm{p}_{4}} A_{n+1}^{y_{r}}, \quad A_{y_{r}}^{n} \subset_{\mathrm{p}_{4}} A_{y_{r}}^{n+1} \text {, }
\end{aligned}
$$

and if $y_{s}<y_{t}$ and $n \geqslant \max (s, t)$, then

3. $\quad A_{n}^{y_{s}} \subset_{\mathrm{p}_{4}} A_{n}^{y_{\mathrm{t}}}, \quad A_{y_{\mathrm{t}}}^{n} \subset_{\mathrm{p}_{4}} A_{y_{s}}^{n}$.

But since $g$ preserves the $\mathrm{p}_{4}$-property, the system of sets $\left\{g\left(A_{n}^{y r}\right), g\left(A_{y_{r}}^{n}\right)\right\}$ is a $\mathrm{p}_{4}$-system for $f \circ g^{-1}$. By Theorem 5 (see [2]), $f \circ g^{-1} \in \mathscr{M}_{4}$ if and only if there exists $\mathrm{p}_{4}$-system for $f_{\circ} g^{-1}$. Then $f_{\circ} g^{-1} \in \mathscr{M}_{4}$ and $g^{-1} \in H_{4}$. Suppose that $g^{-1} \in H_{4}$. At first we show that if $A$ is a closed set, $B$ is a perfect set, $A \subset B$, and $\sup \left\{d_{4}\left(B^{\prime}, x\right)\right\}<1-\eta<1$ then $g(A) \subset{ }_{\mathrm{p}_{4}} g(B)$.

Let $E=B_{1} \cup A$, where $B_{1}$ is a set of type $F_{\sigma},\left|B_{1}\right|=|B|, B_{1} \subset B$ and every point of $B_{1}$ is a point of density $B_{1}$. It follows from the inequality $d_{4}\left(E^{\prime}, x\right)<1-\eta$ for every $x \in A$, that $E \in M_{4}$. From Lemma 1 we have $g(E) \in M_{4}$. By Lemma 2 there exists a sequence $\left\{F_{n}\right\}_{n=1}^{\infty}$ of closed sets and a sequence $\left\{\eta_{n}\right\}_{n=1}^{\infty}$ of positive numbers such that $g(E)=\bigcup_{n=1}^{\infty} F_{n}$ and for every $n$ and $y \in F_{n} d_{4}\left(g\left(E^{\prime}\right), \hat{y}\right)<1-\eta_{n}$. 
Thus $g(A)=\bigcup_{n=1}^{\infty} g(A) \cap F_{n}$ and for every $n$ and $y \in g(A) \cap F_{n}$, we obtain $d_{4}\left(g\left(E^{\prime}\right), y\right)<1-\eta_{n}$. From the inclusion $E^{\prime} \supset B^{\prime}$ it follows that $d_{4}\left(g\left(B^{\prime}\right), y\right)<1-\eta_{n}$ and then $g(A) \subset_{\mathrm{p}_{4}} g(B)$. Now let $A, B$ be a pair of perfect sets such that $A \subset \subset_{\mathrm{p}_{4}} B$. By the definition of $\mathrm{p}_{4}$-property we have $A=\bigcup_{n=1}^{\infty} F_{n}$ where $\left\{F_{n}\right\}_{n=1}^{\infty}$ is a sequence of closed sets and there exists a sequence $\left\{\eta_{n}\right\}_{n=1}^{\infty}$ of positive numbers such that for every $n$ and $x \in F_{n}, d_{4}\left(B^{\prime}, x\right)<1-\eta_{n}$. Since $F_{n}$ is closed, we have $g\left(F_{n}\right) \subset_{p_{4}} g(B)$ which gives us that $g(A)=\bigcup_{n=1}^{\infty} g\left(F_{n}\right) \subset_{\mathrm{p}_{4}} g(B)$. This completes the proof of theorem.

THEOREM 3. The homeomorphism classes $\mathrm{H}_{5} \backslash \mathrm{H}_{4}$ and $\mathrm{H}_{4} \backslash \mathrm{H}_{5}$ are nonempty.

Proof. Let $C$ be the Cantor set in $(0,1)$ and let $\left\{G_{l}\right\}_{i=1}^{\infty}=\left\{\left(a_{i}, b_{i}\right)\right\}_{i=1}^{\infty}$ denote the sequence of the interval contiguous to $C$. Define for $i=1,2, \ldots I_{i k}=\left[a_{i}+\right.$ $\left.+2^{-k}-2^{-k}(p+2)^{-1}, a_{i}+2^{-k}\right]$ where $p$ is such that $\left|G_{i}\right|=3^{-p}, k \geqslant k(i)$ and $k(i)$ is such that $2^{-k(i)}<\left(b_{i}-a_{i}\right) / 4$. Let us denote for $i, k$ and $j=0,1, \ldots, k, x_{j}=$ $\inf \left(I_{i k}\right)+j|k| I_{i k} \mid$. Moreover, let for $i=1,2, \ldots$ and $k>k(i)$

$$
D_{i k}=\bigcup_{s=1}^{k}\left(x_{s-1}, x_{s-1}+\frac{x_{s}-x_{s-1}}{p}\right)
$$

where $p$ is such that $b_{i}-a_{i}=3^{-p}$,

and

$$
H_{i k}=\bigcup_{s=1}^{k}\left(x_{s-1}, x_{s-1}+\frac{x_{s}-x_{s-1}}{2}\right) \text { if } \quad b_{i}-a_{i}<3^{-1}
$$

and

$$
\begin{gathered}
H_{i k}=\bigcup_{s=1}^{k}\left(x_{s-1}, x_{s}\right) \text { if } b_{i}-a_{i}=\frac{1}{3}, \\
D=C \cup \bigcup_{i=1}^{\infty}\left(G_{i} \backslash \bigcup_{k=k(i)}^{\infty} I_{i k}\right) \cup \bigcup_{i=1}^{\infty} \bigcup_{k=k(i)}^{\infty} D_{i k}
\end{gathered}
$$

$$
H=C \cup \bigcup_{i=1}^{\infty}\left(G_{i} \backslash \bigcup_{k=k(i)}^{\infty} I_{i k}\right) \cup \bigcup_{i=1}^{\infty} \bigcup_{k=k(i)}^{\infty} H_{i k} .
$$

For the sake of simplicity of our notation we keep on writing

$$
A_{j}=G_{j} \backslash \bigcup_{k=k(j)}^{\infty} I_{j k} \quad(j=1,2, \ldots)
$$

J. Lipiński in [8] proved that $D \notin M_{4}$.

We show that for every $a \in H, d_{4}\left(H^{\prime}, a\right)<1-\frac{1}{8}$. In the proof we are using the method presented by J. Lipiński in [8]. He proved that for all $a \in C \bigcup_{i=1}^{\infty}\left\{a_{i}, b_{i}\right\}$, $d\left(\bigcup_{i=1}^{\infty} A_{i}, a\right)=1$. By inclusion $\bigcup_{i=1}^{\infty} A_{i} \subset H$, we get $d(H, a)=1$ and by Lemma 3 (ii) $d_{4}\left(H^{\prime}, a\right)=0$. The set

$$
\bigcup_{i=1}^{\infty}\left(G_{i} \backslash \bigcup_{k=k(i)}^{\infty} I_{i k}\right) \cup \bigcup_{i=1}^{\infty} \bigcup_{k=k(i)}^{\infty} H_{i k}
$$

is open and it follows that for all $a$ belonging to this set we have $d(H, a)=1$ and by Lemma 3 (ii), also $d_{4}\left(H^{\prime}, a\right)=0$. It remains to prove that $d_{4}\left(H^{\prime}, a\right)<1-1 / 8$ for $a \in \bigcup_{i=1}^{\infty}\left\{a_{i}, b_{l}\right\}$. J. Lipiński proved also that for all $j=1,2, \ldots$

$$
d^{-}\left(\bigcup_{i=1}^{\infty} A_{i}, a_{j}\right)=1 \quad \text { and } \quad d^{+}\left(\bigcup^{\infty} A_{i}, b_{j}\right)=1
$$

It follows that for all $i=1,2, \ldots$

$$
d^{-}\left(H, a_{i}\right)=1 \text { and } d^{+}\left(H, b_{i}\right)=1 .
$$

Observe that if $I \subset\left(a_{i}, b_{i}\right)$ and $I \cap A_{i} \neq \varnothing$ then

$$
|I \cap H| /|I| \geqslant 1 / 2 \text {. }
$$

We show that for all $i=1,2, \ldots d_{4}\left(H^{\prime}, a_{i}\right)<1-1 / 8$. Let $\left\{I_{n}\right\}_{n=1}^{\infty}$ be a sequence of closed intervals such that $\left\{I_{n}\right\}_{n=1}^{\infty}$ converging to $a_{i}, I_{n} \subset\left(a_{i}, b_{i}\right)$ and there exists $c>0$ such that $\left|I_{n}\right|>c \cdot \operatorname{dist}\left(a_{i}, I_{n}\right)$. Thus, for estimation of the mean density $\left|I_{n} \cap H\right| /\left|I_{n}\right|$ we need only consider the case where for every $n$ there exists $k=k(n)$ such that $I_{n} \subset H_{i k(n)}$.

Indeed, if it was not true then, by conditions (1), (2),

We have inequality

$$
\left|I_{n} \cap H\right| /\left|I_{n}\right| \geqslant \min (1,1 / 2)=1 / 2 \text {. }
$$

so

$$
\operatorname{dist}\left(a_{i}, I_{i k}\right)=2^{-k}-2^{-k}(p+2)^{-1}<\operatorname{dist}\left(a_{i}, I_{n}\right)<\left|I_{n}\right| / c
$$

and

$$
\left|I_{i k}\right|\left(2^{-k}-2^{-k}(p+2)^{-1}\right) /\left|I_{i k}\right|<\left|I_{n}\right| / c
$$

$$
\left|I_{i k}\right| /\left|I_{n}\right|<1 /(c(p+1)) \text {. }
$$

Let $m=m(n)$ be a number of points $x_{s} \in I_{i k(n)}$ included in interval $I_{n}$. Then

Observe that

$$
\frac{\left|I_{n} \cap H_{i k}\right|}{\left|I_{n}\right|} \geqslant \frac{(m-1) / 2 k\left|I_{i k}\right|}{\left|I_{i k}\right|(m+1) / k}=\frac{1}{2} \frac{(m-1)}{(m+1)}
$$

Then

$$
(m-1) / k\left|I_{i k}\right| \leqslant\left|I_{n}\right|<(m+1) / k\left|I_{i k}\right| .
$$

$$
\frac{m-1}{m+1} \geqslant \frac{k\left|I_{n}\right| /\left|I_{i k}\right|-2}{k\left|I_{n}\right| /\left|I_{i k}\right|+2}
$$

5 - Fundamenta Mathematicae CXXv. 1 
and

$$
\frac{\left|I_{n} \cap H_{i k}\right|}{\left|I_{n}\right|} \geqslant \frac{1}{2} \frac{k-2 /(c(p+1))}{k+2 /(c(p+1))} .
$$

Since the sequence $\left\{I_{n}\right\}_{n=1}^{\infty}$ converges to $a_{i}, k(n) \rightarrow \infty$. Thus, there exists $N$ such that for every $n \geqslant N$

$$
\left|I_{n} \cap H_{i k(n)}\right| /\left|I_{n}\right| \geqslant 1 / 8 \text {. }
$$

Hence $\varliminf_{n \rightarrow \infty}\left|I_{n} \cap H\right| /\left|I_{n}\right| \geqslant 1 / 8$ so $\varlimsup_{n \rightarrow \infty}\left|I_{n} \cap H^{\prime}\right| /\left|I_{n}\right| \leqslant 1-1 / 8$, and consequently $d_{4}\left(H^{\prime}, a_{i}\right) \leqslant 1-1 / 8$.

By the same estimation for points $b_{i}(i=1,2, \ldots)$ and (1), (2) we have then for $a \in H d_{4}\left(H^{\prime}, a\right) \leqslant 1-1 / 8$,

$$
\begin{aligned}
& \text { Define } g(x)=x \text { for } x \in(1 / 3,2 / 3) \cup C \cup \bigcup_{i=1}^{\infty}\left(G_{i} \backslash \bigcup_{k=k(i)}^{\infty} I_{i k}\right), \\
& g\left(x_{s}\right)=x_{s}(s=0,1,2, \ldots, k), \quad g\left(x_{s-1}+\left(x_{s}-x_{s-1}\right) / 2\right)=x_{s-1}+\left(x_{s}-x_{s-1}\right) / p
\end{aligned}
$$

where $p \geqslant 2, x_{s} \in I_{i k} \subset G_{i}$ and $\left|G_{i}\right|=3^{-p}$ and $g$ is linear on the contiguous intervals. It is easy to verify that $g(H)=D$. By Lemma $1 h=g^{-1} \notin H_{4}$. To show that $h \in H_{5}$ we now prove that $g$ fulfils the hypothesis of Theorem 5 [4], that is $g$ is absolutely continuous homeomorphism, $g^{\prime}$ is essentially bounded and for every $x_{0} \in(0,1)$ there exists $n \in N$ such that $x_{0}$ is the point of dispersion of the set $\left\{x: g^{\prime}(x)<1 / n\right\}$.

If $x_{0} \in G_{i}$, where $\left|G_{i}\right|=3^{-p}$, we take $n$ greater than $p$ and we have

$$
\left\{x: g^{\prime}(x)<1 / n\right\} \cap G_{i}=\varnothing \text {, so } d\left(\left\{x: g^{\prime}(x)<1 / n\right\}, x_{0}\right)=0 \text {. }
$$

Let $x_{0} \in C \backslash \bigcup_{i=1}^{\infty} \bar{G}_{i}$. Since $\left\{x: g^{\prime}(x)<1\right\} \subset(0,1) \backslash \bigcup_{i=1}^{\infty} A_{i}, d\left(\left\{x: g^{\prime}(x)<1\right\}, x_{0}\right)=0$.

The case where $x_{0}$ equals $a_{i}$ or $b_{i}$ is obviously reduced to a combination of the preceding cases. By the definition of the homeomorphism $g, g^{\prime} \leqslant 2$ on the set of its existence, that $g^{\prime}$ is essentially bounded. On the intervals contiguous to Cantor set homeomorphism $g$ is intervals linear, so $g$ is absolutely continuous, hence applying Theorem 5 [4], we get the desired result.

Now we construct an example of a homeomorphism which is in $H_{4} \backslash H_{5}$. Let $x \in(0,1)$. Let for $n \geqslant n_{0}$ where $1 / n_{0}+x<1, p_{n}=1 / n+x, q_{n}=p_{n}+r_{n}$, where $r_{n}$ $=(1 /(n-1)-1 / n) n /(n+1)$. Let $A=(0, x] \cup \bigcup_{n=n_{0}}^{\infty}\left(p_{n}, q_{n}\right)$. Then $d(A, x)=1$. Now, let $q_{n}^{\prime}=\left(p_{n}+q_{n}\right) / 2 \quad\left(n \geqslant n_{0}\right)$, and define the homeomorphism by the following formulae $g(t)=t$, for $0<t \leqslant x, g\left(p_{n}\right)=p_{n}, g\left(q_{n}\right)=q_{n}^{\prime}$ for all $n \geqslant n_{0}$, and $g$ is linear on each interval $\left(p_{n}, q_{n}\right)$ or $\left(q_{n}, p_{n-1}\right)$. From Lemma 1 we conclude that $h=g^{-1} \notin H_{5}$. To prove that $h \in H_{4}$ we use Lemma 1. Let $E \in M_{4}$ and $x \notin E$. We have $g(E)$ $\bigcup_{n=1}^{\infty} g\left(F_{n}\right)$, where $\left\{F_{n}\right\}_{n=1}^{\infty}$ is a sequence of closed sets and for every $n$ and $x_{0} \in F_{n}$. $d_{4}\left(E^{\prime}, x_{0}\right)<1-\eta_{n}$, where $\eta_{n}$ is positive for all $n \in N$.
If $\left\{I_{n}\right\}_{n=1}^{\infty}$ is a sequence of closed intervals converging to $y_{0}$ and $\left|I_{n}\right| / \operatorname{dist}\left(y_{0}, I_{n}\right)$ $>c(c>0)$, then for $j \geqslant j_{0}\left|g\left(E^{\prime}\right) \cap I_{j}\right| /\left|I_{j}\right|=\left|E^{\prime} \cap J_{j}\right| / \mid J_{j}$, where $J_{j}=g^{-1}\left(I_{j}\right)$, and $\left|J_{j}\right|>c \cdot \operatorname{dist}\left(x_{0}, J_{j}\right)$, where $x_{0}=g^{-1}\left(y_{0}\right)$. This follows from the linearity of the homeomorphism $g$ on each side of the point $x_{0}$.

Hence

$$
\varlimsup_{n \rightarrow \infty} \frac{\left|g\left(E^{\prime}\right) \cap I_{j}\right|}{\left|I_{j}\right|}=\varlimsup_{n \rightarrow \infty} \frac{\left|E^{\prime} \cap J_{j}\right|}{\left|J_{j}\right|}<1-\eta_{n} .
$$

Thus for every $y_{0} \in g\left(F_{n}\right), d_{4}\left(g\left(E^{\prime}\right), y_{0}\right)<1-\eta_{n}$ and Lemma 2 implies that $g(E) \in M_{4}$. Now, let $x \in E$. We need only to show that there exists $\eta>0$ such that $d_{4}\left(g\left(E^{\prime}\right), x\right)<1-\eta$. Since $E \in M_{4}$, there exists $\eta_{x}>0$ such that $d_{4}\left(E^{\prime}, x\right)<1-\eta_{x}$. Suppose $\left\{I_{n}\right\}_{n=1}^{\infty}$ is a sequence of closed intervals converging to $x$ and there exists $c>0$ such that $\left|I_{n}\right|>c \cdot \operatorname{dist}\left(x, I_{n}\right)$.

We will show that there exist $n_{0}$ and $c_{1}>0$ such that for all $n>n_{0}\left|J_{n}\right|$ $>c_{1} \cdot \operatorname{dist}\left(x, J_{n}\right)$, where $I_{n}=g^{-1}\left(J_{n}\right),(n=1,2, \ldots)$. Let $I_{n}=\left[a_{n}, b_{n}\right], n \in N$. Then $\left|I_{n}\right|>c \cdot \operatorname{dist}\left(x, I_{n}\right)$ if and only if $b_{n}-a_{n}>c\left(a_{n}-x\right)$ (we assume that $x<a_{n}$ for all $n$ ). Then there exist $k=k(n), s=s(n)$ such that

$$
\inf \left(J_{n}\right) \in\left[p_{s(n)+1}, p_{s(n)}\right), \quad \sup \left(J_{n}\right) \in\left(p_{s(n)-k(n)+1}, p_{s(n)-k(n)}\right] .
$$

By the estimation

$$
1 /(s+1)+x<a_{n}<1 / s+x, \quad 1 /(s-k+1)+x<b_{n}<1 /(s-k)+x
$$

we have

$$
\frac{b_{n}-a_{n}}{a_{n}-x}<\frac{1 /(s-k)-1 /(s+1)}{1 /(s+1)}=\frac{k+1}{s+1}
$$

Then

$$
\varliminf_{n \rightarrow \infty}(k(n)+1) /(s(n)-k(n)) \geqslant c
$$

The sequence $\left\{I_{n}\right\}_{n=1}^{\infty}$ converges to $x$, then this inequality implies that $k(n), s(n)$ converge to $\infty$. Thus there exist $c_{1}>0$ and $n_{0}$ such that

$$
\frac{\left|J_{n}\right|}{\operatorname{dist}\left(x, J_{n}\right)} \geqslant \frac{\left|\left(p_{s}, p_{s-k+1}\right)\right|}{\left|\left(x, p_{s}\right)\right|}=\frac{k-1}{s-k+1}>c_{1} \text {. }
$$

Next, we estimate the expression $\left|I_{n} \cap g(E)\right| /\left|I_{n}\right| \cdot g^{\prime} \geqslant 1 / 2$ at every point which exists, so

$$
\left|I_{n} \cap g(E)\right| /\left|I_{n}\right|=\left|g\left(J_{n} \cap E\right)\right| /\left|I_{n}\right| \geqslant \frac{1}{2}\left|E \cap J_{n}\right| /\left|J_{n}\right| \cdot\left|J_{n}\right| /\left|I_{n}\right|
$$

We estimate expression $\left|J_{n}\right| /\left|I_{n}\right|$.

First we can see that

$$
\frac{d\left(g^{-1}\right)}{d u}(u)=2 \text { for } u \in\left(p_{n}, q_{n}^{\prime}\right)
$$


and

$$
\frac{d\left(g^{-1}\right)}{d u}(u)=\frac{1}{n+3} \quad \text { for } \quad u \in\left(q_{n+1}^{\prime}, p_{n}\right)
$$

Then we have

$$
\begin{aligned}
\left|I_{n}\right|-\left|J_{n}\right|= & \left|I_{n} \cap\left(p_{s+1}, q_{s+1}^{\prime}\right)\right|-\left|g^{-1}\left(I_{n} \cap\left(p_{s+1}, q_{s+1}^{\prime}\right)\right)\right|+ \\
& +\left|I_{n} \cap\left(q_{s+1}^{\prime}, p_{s}\right)\right|-\left|g^{-1}\left(I_{n} \cap\left(q_{s+1}^{\prime}, p_{s}\right)\right)\right|+ \\
& +\left|I_{n} \cap\left(P_{s-k+1}, q_{s-k+1}^{\prime}\right)\right|-\left|g^{-1}\left(I_{n} \cap\left(p_{s-k+1}, q_{s-k+1}^{\prime}\right)\right)\right|+ \\
& +\left|I_{n} \cap\left(q_{s-k+1}^{\prime}, p_{s-k}\right)\right|-\left|g^{-1}\left(I_{n} \cap\left(q_{s-k+1}^{\prime}, p_{s-k}\right)\right)\right| \\
\leqslant & (1-2 /(s+3))\left|I_{n} \cap\left(q_{s+1}^{\prime}, p_{s}\right)\right|+(1-2 /(s-k+3))\left|I_{n} \cap\left(q_{s-k+1}^{\prime}, p_{s-k}\right)\right| \\
\leqslant & r_{s+1} / 2+r_{s-k+1} / 2 .
\end{aligned}
$$

That is,

$$
\left|J_{n}\right| /\left|I_{n}\right| \geqslant 1-\frac{1}{2}\left(r_{s+1} /\left|I_{n}\right|+r_{s-k+1} /\left|I_{n}\right|\right) .
$$

By the estimation $\left|I_{n}\right|$ and the definition $r_{n}$ we see that

$$
\frac{r_{s+1}}{\left|I_{n}\right|} \leqslant \frac{s-k+1}{(k-1)(s+1)(s+2)} \quad \text { and } \quad \frac{r_{s-k+1}}{\left|I_{n}\right|} \leqslant \frac{s}{(s-k)(s-k+1)(k-1)} .
$$

Observe that $s=s(n), k=k(n)$ converge to $\infty$ and $s-k>1$. Hence there exists $0<\delta<2$ and $n_{0}$ such that for every $n>n_{0}$

$$
\frac{r_{s+1}}{\left|I_{n}\right|}+\frac{r_{s-k+1}}{\left|I_{n}\right|}<\delta
$$

that is, there exists $\eta_{1}>0$ such that

$$
\frac{\left|J_{n}\right|}{\left|I_{n}\right|}>1-\frac{1}{2} \delta>\eta_{1}, \quad \text { for } \quad n>n_{0} .
$$

The set $E \in M_{4}$. By the definition of $M_{4}$-sets there exists $n_{1}>n_{0}$ such that for all $n>n_{1}$

$$
\frac{\left|E \cap J_{n}\right|}{\left|J_{n}\right|}>\eta_{x}
$$

Consequently

$$
\frac{\left|g(E) \cap I_{n}\right|}{\left|I_{n}\right|}>\frac{1}{2} \eta_{x} \eta_{1}, \text { for } n>n_{1} .
$$

Finally, $\lim _{n \rightarrow \infty}\left|g\left(E^{\prime}\right) \cap I_{n}\right| /\left|I_{n}\right| \leqslant 1-\frac{1}{2} \eta_{x} \eta_{1}$ and we obtain the desired inequality with $\eta=\frac{1}{2} \eta_{x} \eta_{1}$.

THEOREM 4. If the homeomorphisms $g$ and $g^{-1}$ satisfy a Lipschitz condition then both $g$ and $g^{-1}$ are in $H_{4}$.
Proof. Let $g$ and $g^{-1}$ fulfil a Lipschitz condition with constans $L$ and $l$ respectively. We show that $g$ fulfils the condition $H$ of Definition 5. Let $A$ and $B$ be a perfect sets, and suppose $A \subset_{\mathrm{p}_{4}} B$. We shall prove that $g(A) \subset_{\mathrm{p}_{4}} g(B)$. We first prove that if $\left\{I_{n\}_{n=1}^{\infty}}^{\infty}\right.$ is a sequence of closed intervals converging to $y$ and there exists a constant $c>0$ such that $\left|I_{n}\right|>c \cdot \operatorname{dist}\left(y, I_{n}\right)$, for $n=1,2, \ldots$, then there exists a constant $c_{1}>0$ such that $\left|J_{n}\right|>c_{1} \cdot \operatorname{dist}\left(x, I_{n}\right)$, where $J_{n}=g^{-1}\left(I_{n}\right)$, $x=g^{-1}(y), n=1,2, \ldots$. Denote $I_{n}=\left[a_{n}, b_{n}\right]$. Since $\left|I_{n}\right|>c \cdot \operatorname{dist}\left(y, I_{n}\right)$, we have $\left(b_{n}-a_{n}\right) /\left(a_{n}-y\right)>c$ for all $n \in N$ (we consider $a_{n}>y$ ).

Let $J_{n}=\left[a_{n}^{\prime}, b_{n}^{\prime}\right], n \in N$. Then

$$
b_{n}-a_{n}=g\left(b_{n}^{\prime}\right)-g\left(a_{n}^{\prime}\right) \leqslant L\left(b_{n}^{\prime}-a_{n}^{\prime}\right)
$$

and

$$
\frac{b_{n}^{\prime}-a_{n}^{\prime}}{a_{n}^{\prime}-x} \geqslant \frac{1}{L} \frac{b_{n}-a_{n}}{a_{n}-y} \frac{a_{n}-y}{a_{n}^{\prime}-y} \geqslant \frac{1}{L} c \frac{1}{l}=\frac{c}{L l}
$$

Thus

$$
\left|J_{n}\right|>c_{1} \cdot \operatorname{dist}\left(x, J_{n}\right), \quad \text { where } \quad c_{1}=c /(L \cdot l)
$$

Let $A=\bigcup_{n=1}^{\infty} F_{n}$ where $\left\{F_{n}\right\}_{n=1}^{\infty}$ is a sequence of closed sets and for every $x \in F_{n}$ $d_{4}\left(B^{\prime}, x\right)<1-\eta_{n}$, where $\eta_{n}>0$. Let $\left\{I_{n}\right\}_{n=1}^{\infty}$ be a sequence of closed intervals converging to $y$ such that $\left|I_{n}\right|>c \cdot \operatorname{dist}\left(y, I_{n}\right)$ with $c>0$, then we have

$$
\frac{\left|g(B) \cap I_{n}\right|}{\left|I_{n}\right|}=\frac{\left|g(B) \cap g\left(J_{n}\right)\right|}{\left|g\left(J_{i}\right)\right|}>\frac{1}{L} \frac{1}{l} \frac{\left|B \cap J_{n}\right|}{\left|J_{n}\right|} \text {, where } J_{n}=g^{-1}\left(I_{n}\right) .
$$

Since $\left|J_{n}\right|>c_{1} \cdot \operatorname{dist}\left(x, J_{n}\right), \varlimsup_{n \rightarrow \infty}\left|B^{\prime} \cap J_{n}\right| /\left|J_{n}\right|<1-\eta_{n}$ and thus $\varlimsup_{n \rightarrow \infty}\left|g\left(B^{\prime}\right) \cap I_{n}\right| /\left|I_{n}\right|$ $<1-\frac{1}{L} \frac{1}{l} \eta_{n}$. This completes the proof of the inclusion $g(A) \subset_{\mathrm{p}_{4}} g(B)$. By Theorem 3 we have $g^{-1} \in H_{4}$.

The proof that $g \in H_{4}$ is similar.

PRoposition. Let bA denote the class of bounded derivatives on $(0,1)$. Let $H_{b \Delta}$ be the class of homeomorphisms such that $f_{0} h \in b \Delta$ for every bounded derivative $f$. Then $\mathrm{H}_{b \Delta} \subset \mathrm{H}_{4}$ and the inclusion is proper.

Proof. Let $h \in H_{b \Delta}$ and $E \in M_{4}$. By Theorem 2.6 ([3], p. 96) there exist $f \in B \Delta$ such that $E=\{y: f(y)>0\}$. If $g=h^{-1}$ then $g(E)=\{x: f(h(x))>0\}$. Let $\alpha \in R$. By Theorem $2.5[3] E^{\alpha}(f \circ h) \in M_{4}$ thus $g(E)=E^{0}(f \circ h) \in M_{4}$ and so $g^{-1}$ $=h \in H_{4}$.

A. M. Bruckner in [5] gave the example of a homeomorphism $h$ such that and $h^{-1}$ satisfy Lipschitz condition but $h \notin H_{b \Delta}$. Thus, by Theorem $4, h$ is the homeomorphism such that $h \in H_{4}$ and $h \notin H_{b \Delta}$, so the inclusion is proper. 


\section{References}

[1] S. J. Agronsky, Characterizations of certain subclasses of the Baire class 1, Doctoral Dissertation, University of California, Santa Barbara, 1974.

[2] S. J. Agronsky, A generalization of theorem of Maximoff and applications, to appear.

[3] A. M. Bruckner, Differentiation of Real Functions, Springer-Verlag 1978.

[4] A. M. Bruckner, Density preserving homeomorphisms and a theorem of Maximoff, Quart. J. Math. Oxford Ser. (2) 21 (1970), pp. 337-347.

[5] A. M. Bruckner, On transformations of derivatives, Proc. Amer. Math. Soc., 48 (1975), pp. 101-107.

[6] M. Laczkovich, Separation of sets by bounded derivatives, Period. Math. Hung. Vol. 7 (2), 1976, pp. 169-177.

[7] M. Laczkovich, G. Petruska, On the transformers of derivatives, Fund. Math. 100 (1978), pp. 178-199.

[8] J. Lipiński, Sur certains problèmes de Choquet et de Zahorski concernant les fonctions dérivées, Fund. Math. 44 (1965), pp. 94-102.

[9] Z. Zahorski, Sur la première dérivée, Trans. Amer. Math. Soc. 69 (1950), pp. 1-54.

INSTYTUT MATEMATYKI UNIWERSYTETU GDAŃSKIEGO

INSTITUTE OF MATHEMATICS, UNIVERSITY OF GDAŃSK

Received 4 July 1983

\section{Products of Baire topological vector spaces}

by

\section{Valdivia (Valencia)}

Abstract. Let $I$ be a set whose cardinal number is larger than $\aleph_{0}$. In this article it is proved that there are dense subspaces of $c_{0}(I)$ with additional properties which are Baire and whose product is not Baire. The same properties are obtained taking $l^{p}(I), 0<p<\infty$, instead of $c_{0}(I)$.

1. Introduction. All the linear spaces we shall use are defined over the field $K$ of real or complex numbers. If $E$ is a set, we denote by $E^{\omega}$ the countable infinite product of copies of $E$.

Oxtoby [5] proved that the continuum hypothesis implies that there is a completely regular Baire topological space whose square is not Baire. Actually, Oxtoby uses the hypothesis that the union of $<2^{\mathrm{N}_{0}}$ subsets of Lebesgue measure zero of real numbers has Lebesgue measure zero. P. E. Cohen [2], using forcing techniques, gave an absolute example of Baire spaces whose product is not Baire. Later Fleissner and Kunen [3] gave new examples of Baire spaces whose products is not Baire space without using additional hypothesis of the theory of sets. In this article we give examples of Baire topological vector spaces whose product is not Baire using in part techniques of Fleissner and Kunen [3].

Given a set $I$ and an ordinal $\alpha$ we denote by card $I$ and $\operatorname{card} \alpha$ the cardinal numbers of $I$ and $\alpha$ respectively. If $\beta<\alpha,[\beta, \alpha[$ is the interval of ordinal numbers closed in $\beta$ and open in $\alpha$, i.e.,

$$
[\beta, \alpha[=\{\delta: \beta \leqslant \delta<\alpha\} .
$$

We represent by $\omega_{1}$ the first ordinal such that card $\omega_{1}>\aleph_{0}$. We suppose $[0, \alpha]$ endowed with the order topology. A subset of $[0, \alpha[$ is said to be stationary if it meets every unbounded closed subset of $[0, \alpha]$. Let $\gamma$ be the first ordinal such that card $I=\operatorname{card} \gamma$ and let $T_{n}$ be a mapping from $[0, \gamma[$ in $[0, \gamma[, n=1,2, \ldots$ We shall need the following results:

(a) If card $\gamma>N_{0}$ the set

$$
\left\{\alpha<\gamma: T_{n}([0, \alpha \mathrm{D} \subset[0, \alpha[, n=1,2, \ldots\}\right.
$$

is unbounded and closed in $[0, \gamma[$, 